\title{
Identification of Heterotic Crosses for yield and Water Use Efficiency Traits in Relation to Moisture Stress Tolerance in Groundnut (Arachis hypogaea L.)
}

\author{
K. John ${ }^{1^{*}}$, P. Raghava Reddy ${ }^{2}$, T. C. M. Naidu ${ }^{3}$ and N. V. Naidu ${ }^{4}$
}

${ }^{1}$ Dept. of Genetics and Plant Breeding, Institute of Frontier Technology, Regional Agricultural Research Station, Tirupati, Andhra Pradesh (517 502) India

${ }^{2}$ Dept. Genetics and Plant Breeding, ${ }^{4}$ Dept. of Genetics and Plant Breeding, Acharya N. G. Ranga Agricultural University, Andhra Pradesh (522 034), India

${ }^{3}$ Dept. of Crop Physiology, Institute of Frontier Technology, Regional Agricultural Research Station, Tirupati, Andhra Pradesh (517 502), India

\section{Corresponding Author}

K. John

e-mail: johnlekhana@rediffmail.com

\author{
Article History \\ Article ID: 3 C0406 \\ Received in 07 ${ }^{\text {th }}$ October, 2017 \\ Received in revised form $29^{\text {th }}$ June, 2018 \\ Accepted in final form $25^{\text {th }}$ July, 2018
}

\begin{abstract}
The experimental material comprised of $24 \mathrm{~F}_{1}$ crosses generated through LinexTester analysis. The relative heterosis, heterobeltiosis and standard heterosis were estimated for yield and water use efficiency traits. The heterobeltiosis for SPAD chlorophyll meter reading ranged from -20.45\% (K-6xICGV-91114) to 15.25\% (TAG-24×TMV-2). For specific leaf area the heterobeltiosis ranged from 41.46\% (TAG24×ICGV-91114) to $17.03 \%$ (RohinixDharani). The cross PrasunaxTCGS -1416 recorded the highest heterobeltiosis for number of mature pods per plant (53.33\%), RohinixICGV-91114 for shelling per cent (8.58\%), PrasunaxDharani for sound mature kernel per cent (10.04\%), TAG-24×Dharani for kernel yield plant ${ }^{-1}(90.75 \%)$ and PrasunaxTCGS-1416 for pod yield plant ${ }^{-1}(60.69 \%)$. Based on heterobeltiosis the best heterotic crosses were identified for different traits viz., SPAD chlorophyll meter reading (TAG-24×TMV-2, K-6×TMV-2 and Greeshma x TMV-2), specific leaf area (TAG-24×ICGV-91114, K-6×TMV-2 and GreeshmaxTCGS-1416), shelling per cent (Rohini x ICGV-91114, K-6×TCGS-1416 and TAG-24×Dharani), kernel yield plant ${ }^{-1}$ (TAG-24×Dharani, GreeshmaxDharani and GreeshmaxTMV-2) and pod yield plant ${ }^{-1}$ (Prasuna×TCGS-1416, TAG-24×Dharani and PrasunaxICGV-91114). These hybrids offer best possibilities of future exploitation for development of high yielding moisture stress tolerant groundnut genotypes.
\end{abstract}

Keywords: Heterosis, yield, water use efficiency traits, groundnut

\section{Introduction}

Water use efficiency is the ratio of the total drymatter produced per unit of water transpired. Variation in WUE among genotypes of same species was first documented by Briggs and Shantz (1913) and the possibility of using this as a selection trait in breeding for drought tolerant genotypes has been reported by Tanner and Sinclair (1983). Recent studies have confirmed that substantial genotypic variation for WUE exists in groundnut (Hubick et al., 1986). Wright et al. (1988) showed that the genotypic variability for WUE in groundnut ranged from 2.15 to $3.71 \mathrm{~g}$ of dry matter per $\mathrm{kg}$ of water used. Hebbar (1990) reported a variation from 1.57 to $2.66 \mathrm{~g}$ drymatter per kg of water. Wright et al. (1993) reported a genotypic variation from 1.8 to $3.7 \mathrm{~g}$ of dry matter production per kg of water used. Genotypic differences in WUE of 4 peanut genotypes ranged from 1.81 to $3.05 \mathrm{~g} \mathrm{~kg}^{-1}$ under intermittent water deficit and from 2.07 to $3.15 \mathrm{~g} \mathrm{~kg}^{-1}$ under continuous water deficit. These studies suggest the possibility of using this trait in breeding for drought resistance.

Selection for higher WUE often resulted in decrease in crop growth rates and this was perhaps the most significant setback for further improvement in this trait. This lack of success arises primarily due to strong inter-dependency between transpiration and WUE. Therefore it is essential to identify types where this interdependency is lower. According to Udayakumar et al. (1998), two important physiological traits that determine the variability in WUE are photosynthetic rate and transpiration rate. Photosynthetic rate is regulated by intrinsic mesophyll efficiency and the $\mathrm{CO}_{2}$ diffusive process associated with stomata. Transpiration rate is predominantly controlled by differences in mesophyll efficiency. They stated that in crops like groundnut (capacity type) WUE is independent of total transpiration and selection for WUE is likely to result in higher total drymatter production. Water 
use efficiency is known to increase with decrease in the quantity of water applied without substantial decrease in yield (Hammond and Boote, 1981). Nimitr Vorasoot et al. (1988) found WUE to be higher in groundnuts that received lower water regimes at longer irrigation intervals ( 14 days). Rosario and Fajardo (1988) reported that WUE decreased under water stress in all cultivars of groundnut. Genotypes with greater decrease in stomatal conductance and a minimum change in assimilation rate always show a greater increase in WUE under stress (Hebbar, 1990). Significant positive relation between WUE values obtained in control and stress treatments was also reported by many workers indicating that the GXE is very low for this trait (Wright et al., 1992). Even though WUE was high under stress, the genotypic ranking has been shown to remain constant under non stress and stress conditions (Udayakumar et al., 1998). Hebbar et al. (1994) showed that more than $92 \%$ of the variation in dry matter accumulation was accounted by the variation in WUE. WUE varied significantly between genotypes and there was a positive correlation between WUE and the quantity of dry matter produced by the genotypes. Babitha and Reddy (2001) reported that total drymatter produced per plant had positive correlation with WUE in simulated drought $(r=0.31, p<0.05)$ and rain fed treatments $(r=0.42, p<0.05)$ and water use efficient genotypes were high TDM producers with poor harvest index.

Most of the groundnut breeding programmes aimed at improving productivity have been directed towards hybridization followed by selection in segregating generation. Since groundnut is a predominately self pollinated crop and commercial product of $F_{1}$ seed is not currently feasible, it was felt that heterosis in groundnut is unstable. However, the magnitude of heterosis provide the basis of genetic diversity and a guide for choice of desirable parents for developing superior $\mathrm{F}_{1}$ hybrids to exploit hybrid vigour and are building gene pool to be employed in breeding programme. Heterosis in $F_{1}$ generation expressed in terms of superiority over the better/mid-parent/standard parent is of direct relevance not only for developing hybrids in cross-pollinated crops, but also in self pollinated crops because heterotic crosses help the breeder to select appropriate crosses which would lead to desirable transgressive segregants in advanced generations (Arunachalam et al., 1984). Groundnut is a highly self pollinated crop and the scope for exploitation of hybrid vigour will depend on the direction and magnitude of heterosis, biological and feasibility and nature of gene action. In groundnut, heterosis cannot be exploited for higher production through commercial hybrids due to cleistogamous nature of flower and poor seed recovery during hybridization. For the development of an effective heterosis breeding programme in groundnut, one need to have information about genetic architecture and estimated prepotency of parents in hybrid combinations (Waghmode et al., 2017). Study of heterosis will have a direct bearing on the breeding methodology to be employed for varietal improvement. Therefore, the present study was planned to estimate the extent of heterosis over mid parent, better parent and standard parent in twenty four $\mathrm{F}_{1} \mathrm{~s}$ for yield and water use efficiency traits.

\section{Materials and Methods}

The experimental material comprised of twenty four $F_{1}$ single crosses generated through LinexTester analysis using six lines viz., TAG-24, Prasuna, Rohini, Narayani, K-6 and Greeshma and four testers viz., Dharani, TMV-2, TCGS-1416 and ICGV-91114. These were grown in a randomized block design with three replications during kharif 2014 at Regional Agricultural Research Station, Tirupati. The $F_{1}$ hybrids were grown in plots consisting of single row of $5 \mathrm{~m}$ length having a spacing of $30.0 \times 10 \mathrm{~cm}^{2}$. In parents and $F_{1}$ hybrids, twenty plants per replication per genotype per replication per cross were sampled for recording observations. Data were recorded for plant height $(\mathrm{cm})$, number of primary branches plant $^{-1}$, no. of secondary branches plant ${ }^{-1}$, SPAD Chlorophyll meter reading, specific leaf area $\left(\mathrm{cm}^{2} \mathrm{~g}^{-1}\right)$, no. of mature pods plant ${ }^{-1}$, shelling per cent, sound mature kernel per cent, kernel yield plant ${ }^{-1}(\mathrm{~g})$ and pod yield plant ${ }^{-1}(\mathrm{~g})$. The water use efficieny traits viz., SPAD chlorophyll meter reading (SCMR) was measured on all four-leaflets of third leaf from the top on main axis at 60 DAS under normal sunlight using SPAD meter of Minolta Company, NJ, USA (SPAD-502). Specific leaf area (SLA) was recorded at 60 days after sowing. Ten leaves ( $3^{\text {rd }}$ fully expanded leaf from the top on the main axis) were collected from each treatment in each replication for calculating SLA. These leaves were cleaned and their leaf area was estimated using a leaf area meter (LICOR model-3100). They were dried in a hot air oven at $80^{\circ} \mathrm{C}$ and dry weight recorded. The formula used was:

$\mathrm{SLA}=\frac{\text { Leaf area }\left(\mathrm{cm}^{2}\right)}{\text { Leaf dry weight }(\mathrm{g})}$

The overall mean values of each character for each parent and $F_{1}$ utilized and relative heterosis was calculated as the per cent deviation of mean of the $F_{1}$ from its mid parental value between two corresponding parents. Heterobeltiosis was estimated as difference between the mean of the $F_{1}$ and that of the parent with greater expression for each of the character in each $F_{1}$ combination. Standard heterosis was calculated as the per cent deviation of mean of $F_{1}$ from standard parent. Heterosis over mid parent (relative heterosis), better parent (heterobeltiosis) and standard parent (standard heterosis) in $F_{1}$ generation in each cross were estimated using standard formulae.

Relative heterosis (\%) $=\frac{\bar{F}_{1}-\bar{M}_{p}}{\bar{M}_{p}} \times 100$
Heterobeltiosis (\%) $=\frac{\bar{F}_{1}-\bar{B}_{p}}{\bar{B}_{p}} \times 100$
Standard heterosis (\%) $=\frac{\overline{\bar{F}}_{1}-\bar{S}_{p}}{\overline{S_{p}}} \times 100$ 


\section{Results and Discussion}

Heterosis or hybrid vigour is measured as an increase or decrease of a trait mid-parental value (relative heterosis), over better parent (heterobeltiosis) and standard parent (standard heterosis) for water use efficiency traits and yield are computed and presented in Tables 1 and 2 .

\subsection{Plant height $(\mathrm{cm})$}

Relative heterosis for plant height ranged from -31.13\% (TAG$24 \times T M V-2$ ) to $20.77 \%$ (TAG-24×Dharani). Two $F_{1}$ s exhibited positive significant relative heterosis. Significant negative heterosis over mid-parent was observed in eight $F_{1} s$. The minimum and maximum heterosis for plant height over better parent was observed in $\mathrm{F}_{1} \mathrm{~S}$ TAG-24×TMV-2 (-38.20\%) and TAG-24×Dharani (20.59\%) respectively. Thirteen $\mathrm{F}_{1}$ s exhibited significant negative heterosis over better parent and only one $\mathrm{F}_{1}$ exhibited positive significant heterosis over better parent. Standard heterosis ranged from -36.72\% (TAG-24×TMV-2) to $18.40 \%$ (NarayanixDharani). Significant and negative heterosis over standard parent was observed in seven $\mathrm{F}_{1} \mathrm{~s}$.

\subsubsection{No. of primary branches plant ${ }^{-1}$}

The range of heterosis over mid parent varied from $-20.00 \%$ (TAG-24×Dharani) to 74.17\% (GreeshmaxDharani). Significant positive heterosis was observed in six $\mathrm{F}_{1}$ s only, while significant negative heterosis was noticed in eight $\mathrm{F}_{1} \mathrm{~s}$. Heterobeltiosis for number of primary branches plant ${ }^{-1}$ ranged from $-20.00 \%$ (seven $\mathrm{F}_{1} \mathrm{~s}$ ) to $74.17 \%$ (GreeshmaxDharani). Among twenty four $\mathrm{F}_{1} \mathrm{~s}$ studied, six $\mathrm{F}_{1}$ s exhibited significant positive heterosis over better parent. Negative and significant heterosis was noticed in seven $\mathrm{F}_{1} \mathrm{~s}$ over better parent. Standard heterosis ranged from $-0.00 \%$ (Sixteen $F_{1} s$ ) to $74.17 \%$ (GreeshmaxDharani). Significant and positive heterosis over standard parent was observed in eight $\mathrm{F}_{1} \mathrm{~s}$.

\subsubsection{No. of secondary branches plant ${ }^{-1}$}

The range of heterosis over mid-parent varied from $93.33 \%$ (TAG-24×Dharani) to $1900.00 \%$ (NarayanixICGV-91114 and $\mathrm{K}-6 \times \mid \mathrm{CGV}-91114)$. Out of twenty four $F_{1} s$, eighteen $F_{1} s$ recorded significant positive heterosis over mid-parent and non significant positive heterosis was noticed in two $F_{1}$ s. Significant negative heterosis was noticed in four $F_{1} s$. Heterobeltiosis ranged from $-95.00 \%$ (TAG-24×Dharani) to 1900.00\% (NarayanixICGV-91114 and K-6xICGV-91114). Significant and positive heterosis was observed in ten $F_{1}$ s. Significant negative heterosis was noticed in four $F_{1} s$. Standard heterosis ranged from $-90.00 \%$ (Four $F_{1}$ s) to $300.00 \%$ (PrasunaxTCGS-1416). Out of twenty four $F_{1} s$, eight $F_{1} s$ recorded significant positive heterosis over standard parent.

\subsubsection{SPAD chlorophyll meter reading}

The range of heterosis over mid parent varied from $17.00 \%$ (K-6×ICGV-91114) to $15.25 \%$ (TAG-24×TMV-2). Significant positive heterosis was observed in three F1s and thirteen $F_{1} S$ recorded significant and negative heterosis over mid-parent.
Positive and non significant heterosis was noticed in four $F_{1} s$ and non significant negative heterosis over better parent was observed in four $\mathrm{F}_{1} \mathrm{~s}$. Heterobeltiosis for SPAD chlorophyll meter reading at 60 days after sowing ranged from $-20.45 \%$ (K-6xICGV-91114) to $15.25 \%$ (TAG-24×TMV-2). Among twenty four F1s studied, three F1s exhibited significant positive heterosis over better parent. Thirteen F1s recorded significant and negative heterosis over better parent. Positive and non significant heterosis was noticed in only two $\mathrm{F}_{1} \mathrm{~s}$ and non significant negative heterosis over better parent was noticed four in four F1s. Standard heterosis ranged from $-12.50 \%$ (K-6×ICGV-91114) to $15.25 \%$ (TAG-24×TMV-2). Significant positive heterosis was observed in three $F_{1}$ s. Significant negative heterosis was observed in nine $F_{1}$ s. Positive and non significant heterosis was noticed in seven $F_{1} s$ and non significant negative heterosis over better parent was noticed four in five F1s. These results are in agreement with the results of Seethala Devi (2004).

\subsubsection{Specific leaf area $\left(\mathrm{cm}^{2} \mathrm{~g}^{-1}\right)$}

The range of heterosis over mid-parent varied from $-28.93 \%$ (TAG-24×ICGV-91114) to $28.42 \%$ (RohinixDharani). Out of twenty four F1s, six F1s recorded significant positive heterosis over mid-parent. Significant negative heterosis was noticed in fifteen $F_{1}$ s. Heterobeltiosis ranged from $41.46 \%$ (TAG$24 \times \mid C G V-91114$ ) to $17.03 \%$ (RohinixDharani). Significant and positive heterosis was observed in seventeen $\mathrm{F}_{1}$ s. Significant negative heterosis was noticed in only three $\mathrm{F}_{1} \mathrm{~s}$. Standard heterosis ranged from $0.68 \%$ (TAG-24×ICGV-91114) to 97.19\% (Rohinix Dharani). Out of twenty four F1s, twenty F1s recorded significant positive heterosis over standard parent and significant negative heterosis was noticed in only one $F_{1}$. Peanut genotypes with low SLA had more photosynthetic machinery and the potential for greater assimilation per unit leaf area and large genotypic differences in the rate of light saturated photosynthesis per unit area was reported by Wright and Bell (1992). Heterosis for SLA in groundnut was reported earlier by Pallas and Samish (1974), Bhagsari and Brown (1976); Pallas (1982).

\subsubsection{No. of mature pods plant ${ }^{-1}$}

The range of heterosis over mid-parent varied from $-16.67 \%$ (NarayanixDharani) to $78.27 \%$ (PrasunaxTCGS-1416). Out of twenty four $F_{1} s$, thirteen $F_{1} s$ recorded significant positive heterosis over mid-parent. Significant negative heterosis was noticed in four $\mathrm{F}_{1}$ s. Heterobeltiosis ranged from $22.62 \%$ (NarayanixDharani) to $53.33 \%$ (PrasunaxTCGS-1416). Significant and positive heterosis was observed in twelve $F_{1}$ s. Significant negative heterosis was noticed in eight $F_{1} s$. Standard heterosis ranged from 1.33\% (RohinixTMV-2) to $114.67 \%$ (PrasunaxTCGS-1416). Out of twenty four $F_{1}$ s, twenty two $F_{1} s$ recorded significant positive heterosis over standard parent and non significant positive heterosis was noticed in only two $F_{1}$ s. Similar results were reported by John et al. (2012). 


\subsubsection{Shelling per cent}

The relative heterosis for shelling per cent varied from -12.36\% (PrasunaxTCGS-1416) to 9.33\% (Rohinix|CGV-91114).
Eight $F_{1} s$ registered significant positive heterosis and eight $\mathrm{F}_{1} \mathrm{~s}$ showed significant negative heterosis over mid parent. Heterobeltiosis ranged from -12.56\% (PrasunaxTCGS-1416)

\begin{tabular}{|c|c|c|c|c|c|c|c|c|c|c|c|c|}
\hline \multirow[t]{3}{*}{ Crosses } & \multicolumn{12}{|c|}{ Heterosis (\%) } \\
\hline & \multicolumn{3}{|c|}{ Plant height $(\mathrm{cm})$} & \multicolumn{3}{|c|}{$\begin{array}{l}\text { No. of primary branches } \\
\text { plant }^{-1}\end{array}$} & \multicolumn{3}{|c|}{$\begin{array}{l}\text { SPAD chlorophyll meter } \\
\text { reading }\end{array}$} & \multicolumn{3}{|c|}{$\begin{array}{l}\text { Specific leaf area } \\
\left(\mathrm{cm}^{2} \mathrm{~g}^{-1}\right)\end{array}$} \\
\hline & $>\mathrm{MP}$ & $>\mathrm{BP}$ & $>\mathrm{SP}$ & $>\mathrm{MP}$ & $>\mathrm{BP}$ & $>\mathrm{SP}$ & $>\mathrm{MP}$ & $>\mathrm{BP}$ & $>\mathrm{SP}$ & $>\mathrm{MP}$ & $>\mathrm{BP}$ & $>\mathrm{SP}$ \\
\hline $\begin{array}{l}\text { TAG- } \\
\text { 24×|CGV-91114 }\end{array}$ & -11.16 & $-19.44^{* *}$ & $-19.44^{* *}$ & $-11.11^{* *}$ & $-20.00^{* *}$ & 0.00 & 1.07 & 0.25 & 1.92 & $-28.93^{* *}$ & $-41.46^{* *}$ & $-9.57^{* *}$ \\
\hline TAG-24×TMV-2 & $-31.13^{* *}$ & $-38.2^{* *}$ & $-36.72^{* *}$ & $11.11^{* *}$ & 0.00 & $25.00^{* *}$ & $15.25^{* *}$ & $15.25^{* *}$ & $17.17^{* *}$ & $-17.23^{* *}$ & $-28.33^{* *}$ & $10.72^{* *}$ \\
\hline $\begin{array}{l}\text { TAG- } \\
24 \times \text { TCGS-1416 }\end{array}$ & -5.41 & $-16.54^{*}$ & -11.20 & $-11.11^{* *}$ & $-20.00^{* *}$ & 0.00 & $-11.02^{* *}$ & $-14.39^{* *}$ & $-5.83^{*}$ & $-3.20^{*}$ & $-20.45^{* *}$ & $22.9^{* *}$ \\
\hline TAG-24×Dharani & $20.77^{* *}$ & $20.59^{*}$ & -1.60 & $-20.00^{* *}$ & $-20.00^{* *}$ & 0.00 & 0.41 & -0.41 & 1.25 & $-19.22^{* *}$ & $-29.83^{* *}$ & $8.41^{* *}$ \\
\hline Prasuna×Dharani & 9.27 & 5.260 & 13.60 & 0.00 & 0.00 & 0.00 & 0.83 & 0.00 & 1.67 & $-14.54^{* *}$ & $-31.08^{* *}$ & $12.46^{* *}$ \\
\hline $\begin{array}{l}\text { Prasunax ICGV- } \\
91114\end{array}$ & -4.15 & -6.60 & 0.80 & $25.00^{* *}$ & $25.00^{* *}$ & $25.00^{* *}$ & -4.10 & -4.10 & -2.50 & $4.72^{* *}$ & $-11.37^{* *}$ & $44.64^{* *}$ \\
\hline PrasunaxTMV-2 & -9.74 & -10.38 & -3.28 & 0.00 & 0.00 & 0.00 & $-5.51^{*}$ & $-9.09^{* *}$ & 0.00 & 2.43 & $-17.58^{* *}$ & $34.49^{* *}$ \\
\hline $\begin{array}{l}\text { Prasunax TCGS- } \\
1416\end{array}$ & -8.91 & $-20.01^{* *}$ & -13.68 & $11.11^{* *}$ & 0.00 & $25.00^{* *}$ & 0.00 & -0.82 & 0.83 & $-14.64^{* *}$ & $-27.53^{* *}$ & $18.26^{* *}$ \\
\hline Rohinix TMV-2 & $-19.13^{* *}$ & $-25.60^{* *}$ & $-25.60^{* *}$ & 0.00 & 0.00 & 0.00 & $-5.49^{*}$ & $-6.67^{*}$ & $-6.67^{*}$ & $22.83^{* *}$ & $12.23^{* *}$ & $35.65^{* *}$ \\
\hline $\begin{array}{l}\text { Rohinix ICGV- } \\
91114\end{array}$ & 3.78 & -5.55 & -3.28 & 0.00 & 0.00 & 0.00 & 0.42 & -1.64 & 0.00 & $19.21^{* *}$ & $15.35^{* *}$ & $39.42^{* *}$ \\
\hline Rohinix Dharani & $-29.5^{* *}$ & $-36.92^{* *}$ & $-32.88^{* *}$ & 0.00 & 0.00 & 0.00 & -2.81 & $-8.33^{* *}$ & 0.83 & $28.42^{* *}$ & $17.03^{* *}$ & $41.45^{* *}$ \\
\hline $\begin{array}{l}\text { Rohinix TCGS- } \\
1416\end{array}$ & 10.14 & 8.57 & -8.80 & $-11.11^{* *}$ & $-20.00^{* *}$ & 0.00 & $-5.65^{*}$ & $-6.83^{*}$ & $-6.83^{*}$ & $13.83^{* *}$ & $10.55^{* *}$ & $33.62^{* *}$ \\
\hline $\begin{array}{l}\text { Narayanix TCGS- } \\
1416\end{array}$ & 1.92 & -5.35 & 10.40 & 0.00 & 0.00 & 0.00 & $-5.53^{*}$ & $-7.50^{*}$ & $-7.50^{*}$ & $-9.44^{* *}$ & $-28.11^{* *}$ & $22.32^{* *}$ \\
\hline $\begin{array}{l}\text { Narayanix ICGV- } \\
91114\end{array}$ & -8.69 & $-14.27^{*}$ & 0.00 & 0.00 & 0.00 & 0.00 & -0.42 & -3.28 & -1.67 & -2.76 & $-19.08^{* *}$ & $37.68^{* *}$ \\
\hline NarayanixTMV-2 & $-13.7^{*}$ & $-17.49^{* *}$ & -3.76 & 0.00 & 0.00 & 0.00 & $-6.07^{*}$ & $-12.12^{* *}$ & -3.33 & -2.37 & $-22.66^{* *}$ & $31.59^{* *}$ \\
\hline NarayanixDharani & $19.45^{* *}$ & 1.51 & $18.40^{*}$ & $-11.11^{* *}$ & $-20.00^{* *}$ & 0.00 & -2.98 & -5.00 & -5.00 & $-17.96^{* *}$ & $-31.52^{* *}$ & $16.52^{* *}$ \\
\hline K-6 x Dharani & $-14.61^{*}$ & $-19.72^{* *}$ & -8.80 & 0.00 & 0.00 & 0.00 & $-5.39^{*}$ & $-5.79^{*}$ & -5.00 & $12.41^{* *}$ & $-10.48^{* *}$ & $51.01^{* *}$ \\
\hline K-6×TCGS-1416 & -8.15 & $-12.68^{*}$ & -0.80 & $25.00^{* *}$ & $25.00^{* *}$ & $25.00^{* *}$ & $-11.11^{* *}$ & $-11.48^{* *}$ & $-10.00^{* *}$ & $-25.72^{* *}$ & $-37.97^{* *}$ & $4.64^{*}$ \\
\hline K-6xICGV-91114 & $-25.88^{* *}$ & $-28.24^{* *}$ & $-18.48^{*}$ & $25.00^{* *}$ & $25.00^{* *}$ & $25.00^{* *}$ & $-17.00^{* *}$ & $-20.45^{* *}$ & $-12.50^{* *}$ & $-9.41^{* *}$ & $-28.01^{* *}$ & $21.45^{* *}$ \\
\hline K-6xTMV-2 & 8.36 & -6.90 & 5.76 & $-11.11^{* *}$ & $-20.00^{* *}$ & 0.00 & $9.54^{* *}$ & $9.09^{* *}$ & $10.00^{* *}$ & $-26.36^{* *}$ & $-38.32^{* *}$ & 4.06 \\
\hline $\begin{array}{l}\text { Greeshmax Dha- } \\
\text { rani }\end{array}$ & 9.33 & 7.36 & 7.36 & $74.17^{* *}$ & $74.17^{* *}$ & $74.17^{* *}$ & $-5.53^{*}$ & $-7.50^{*}$ & $-7.50^{*}$ & $-23.16^{* *}$ & $-37.04^{* *}$ & -1.45 \\
\hline $\begin{array}{l}\text { Greeshmax ICGV- } \\
91114\end{array}$ & $-25.15^{* *}$ & $-27.34^{* *}$ & $-25.60^{* *}$ & $50.00^{* *}$ & $50.00^{* *}$ & $50.00^{* *}$ & $-10.55^{* *}$ & $-13.11^{* *}$ & $-11.67^{* *}$ & $-8.60^{* *}$ & $-21.3^{* *}$ & $23.19^{* *}$ \\
\hline $\begin{array}{l}\text { Greeshmax } \\
\text { TCGS-1416 }\end{array}$ & $-24.26^{* *}$ & $-27.82^{* *}$ & $-23.20^{* *}$ & $50.00^{* *}$ & $50.00^{* *}$ & $50.00^{* *}$ & $-10.12^{* *}$ & $-15.91^{* *}$ & $-7.50^{*}$ & $-23.22^{* *}$ & $-37.22^{* *}$ & -1.74 \\
\hline $\begin{array}{l}\text { Greeshmax } \\
\text { TMV-2 }\end{array}$ & 7.42 & -0.83 & -4.40 & $-11.11^{* *}$ & $-20.00^{* *}$ & 0.00 & $8.09^{* *}$ & $5.83^{*}$ & $5.83^{*}$ & $-15.11^{* *}$ & $-26.67^{* *}$ & $14.78^{* *}$ \\
\hline SEm \pm & 2.57 & 2.96 & 2.96 & 0.02 & 0.02 & 0.02 & 1.00 & 1.16 & 1.16 & 2.33 & 2.69 & 2.69 \\
\hline
\end{tabular}




\begin{tabular}{|c|c|c|c|c|c|c|}
\hline \multirow[t]{3}{*}{ Crosses } & \multicolumn{6}{|c|}{ Heterosis (\%) } \\
\hline & \multicolumn{3}{|c|}{ No. of secondary branches plant ${ }^{-1}$} & \multicolumn{3}{|c|}{ No. of mature pods plant ${ }^{-1}$} \\
\hline & $>\mathrm{MP}$ & $>B P$ & $>S P$ & $>\mathrm{MP}$ & $>\mathrm{BP}$ & $>\mathrm{SP}$ \\
\hline TAG-24×ICGV-91114 & $33.33^{* *}$ & 0.00 & $100.00^{* *}$ & $45.31^{* *}$ & $36.76^{* *}$ & $55.00^{* *}$ \\
\hline TAG-24×TMV-2 & $90.48^{* *}$ & 0.00 & $100.00^{* *}$ & $18.78^{* *}$ & $14.41^{* *}$ & $29.67^{* *}$ \\
\hline TAG-24×TCGS-1416 & $90.48^{* *}$ & 0.00 & $100.00^{* *}$ & $25.87^{* *}$ & $20.00^{* *}$ & $50.00^{* *}$ \\
\hline TAG-24×Dharani & $-93.33^{* *}$ & $-95.00^{* *}$ & $-90.00^{* *}$ & $46.05^{* *}$ & $32.14^{* *}$ & $85.00^{* *}$ \\
\hline Prasuna×Dharani & 0.00 & 0.00 & 0.00 & $13.86^{* *}$ & $13.39^{* *}$ & $14.33^{* *}$ \\
\hline Prasuna×ICGV-91114 & $93.94^{* *}$ & $6.67^{* *}$ & $6.67^{* *}$ & $27.13^{* *}$ & $24.6^{* *}$ & $30.83^{* *}$ \\
\hline PrasunaxTMV-2 & $263.64^{* *}$ & $100.00^{* *}$ & $100.00^{* *}$ & $23.99^{* *}$ & $12.00^{* *}$ & $40.00^{* *}$ \\
\hline Prasuna× TCGS-1416 & $300.00^{* *}$ & $300.00^{* *}$ & $300.00^{* *}$ & $78.27^{* *}$ & $53.33^{* *}$ & $114.67^{* *}$ \\
\hline RohinixTMV-2 & $-90.00^{* *}$ & $-90.00^{* *}$ & $-90.00^{* *}$ & 3.14 & 1.33 & 1.33 \\
\hline RohinixICGV-91114 & $-81.82^{* *}$ & $-90.00^{* *}$ & $90.00^{* *}$ & $26.88^{* *}$ & $21.75^{* *}$ & $27.83^{* *}$ \\
\hline Rohinix Dharani & $81.82^{* *}$ & 0.00 & 0.00 & $32.13^{* *}$ & $17.07^{* *}$ & $46.33^{* *}$ \\
\hline Rohini× TCGS-1416 & $-90.00^{* *}$ & $-90.00^{* *}$ & $-90.00^{* *}$ & 2.75 & $-13.21^{* *}$ & $21.50^{* *}$ \\
\hline NarayanixTCGS-1416 & $263.64^{* *}$ & $100.00^{* *}$ & $100.00^{* *}$ & 4.55 & -4.17 & $15.00^{* *}$ \\
\hline NarayanixICGV-91114 & $1900.00^{* *}$ & $1900.00^{* *}$ & $100.00^{* *}$ & -2.22 & $-8.33^{* *}$ & $10.00^{* *}$ \\
\hline NarayanixTMV-2 & $900.00^{* *}$ & $900.00^{* *}$ & 0.00 & $20.82^{* *}$ & $18.40^{* *}$ & $48.00^{* *}$ \\
\hline Narayani×Dharani & $81.82^{* *}$ & 0.00 & 0.00 & $-16.67^{* *}$ & $-22.62^{* *}$ & $8.33^{*}$ \\
\hline K-6 x Dharani & $81.82^{* *}$ & 0.00 & 0.00 & -2.27 & $-12.71^{* *}$ & $11.00^{* *}$ \\
\hline K-6×TCGS-1416 & $900.00^{* *}$ & $900.00^{* *}$ & 0.00 & 1.08 & $-7.73^{* *}$ & $17.33^{* *}$ \\
\hline K-6xICGV-91114 & $1900^{* *}$ & $1900.00^{* *}$ & $100^{* *}$ & $-11.96^{* *}$ & $-12.71^{* *}$ & $11.00^{* *}$ \\
\hline $\mathrm{K}-6 \times \mathrm{TMV}-2$ & $81.82^{* *}$ & 0.00 & 0.00 & $-10.17^{* *}$ & $-14.29^{* *}$ & $20.00^{* *}$ \\
\hline GreeshmaxDharani & $81.82^{* *}$ & 0.00 & 0.00 & $46.82^{* *}$ & $34.58^{* *}$ & $61.50^{* *}$ \\
\hline GreeshmaxICGV-91114 & $900.00^{* *}$ & $900.00^{* *}$ & 0.00 & $-5.33^{*}$ & $-11.25^{* *}$ & 6.50 \\
\hline GreeshmaxTCGS-1416 & 0.00 & 0.00 & $-90.00^{* *}$ & 2.86 & 0.80 & $26.00^{* *}$ \\
\hline GreeshmaxTMV-2 & $263.64^{* *}$ & $100.00^{* *}$ & $100^{* *}$ & $11.92^{* *}$ & 3.93 & $45.50^{* *}$ \\
\hline SEm \pm & 0.01 & 0.02 & 0.02 & 0.61 & 0.70 & 0.70 \\
\hline
\end{tabular}

Table 1 Continue...

\begin{tabular}{|c|c|c|c|c|c|c|}
\hline \multirow[t]{3}{*}{ Crosses } & \multicolumn{6}{|c|}{ Heterosis (\%) } \\
\hline & \multicolumn{3}{|c|}{ Shelling per cent } & \multicolumn{3}{|c|}{ Sound mature kernel per cent } \\
\hline & $>\mathrm{MP}$ & $>B P$ & $>S P$ & $>M P$ & $>\mathrm{BP}$ & $>\mathrm{SP}$ \\
\hline TAG-24×ICGV-91114 & $3.93^{* *}$ & 0.90 & 0.900 & $5.01^{* *}$ & $3.66^{* *}$ & $6.39^{* *}$ \\
\hline TAG-24×TMV-2 & 0.21 & 0.00 & $-5.43^{* *}$ & $3.61^{* *}$ & 0.00 & $2.63^{* *}$ \\
\hline TAG-24×TCGS-1416 & 1.41 & 0.00 & $-3.14^{* *}$ & $2.78^{* *}$ & $2.20^{*}$ & $4.89^{* *}$ \\
\hline TAG-24×Dharani & $6.02^{* *}$ & $3.15^{* *}$ & $2.69^{* *}$ & $4.97^{* *}$ & $4.40^{* *}$ & $7.14^{* *}$ \\
\hline Prasuna×Dharani & $-5.83^{* *}$ & $-5.83^{* *}$ & $-5.83^{* *}$ & $10.65^{* *}$ & $10.04^{* *}$ & $11.28^{* *}$ \\
\hline Prasunax|CGV-91114 & $-3.66^{* *}$ & $-6.28^{* *}$ & $-6.28^{* *}$ & 0.96 & $-1.86 *$ & -0.75 \\
\hline Prasuna×TMV-2 & -1.14 & $-2.69^{* *}$ & $-2.69^{* *}$ & $-4.25^{* *}$ & $-4.41^{* *}$ & $-3.01^{* *}$ \\
\hline Prasuna×TCGS-1416 & $-12.36^{* *}$ & $-12.56^{* *}$ & $-12.56^{* *}$ & $-2.04^{*}$ & $-2.22^{*}$ & -0.75 \\
\hline RohinixTMV-2 & $3.02^{* *}$ & -0.45 & -0.45 & $5.90^{* *}$ & $3.99^{* *}$ & $7.89^{* *}$ \\
\hline RohinixICGV-91114 & $9.33^{* *}$ & $8.58^{* *}$ & $2.69^{* *}$ & $7.55^{* *}$ & $3.26^{* *}$ & $7.14^{* *}$ \\
\hline
\end{tabular}




\begin{tabular}{|c|c|c|c|c|c|c|}
\hline \multirow[t]{3}{*}{ Crosses } & \multicolumn{6}{|c|}{ Heterosis (\%) } \\
\hline & \multicolumn{3}{|c|}{ Shelling per cent } & \multicolumn{3}{|c|}{ Sound mature kernel per cent } \\
\hline & $>\mathrm{MP}$ & $>\mathrm{BP}$ & $>\mathrm{SP}$ & $>\mathrm{MP}$ & $>\mathrm{BP}$ & $>\mathrm{SP}$ \\
\hline RohinixDharani & $2.22^{* *}$ & 0.32 & $-2.83^{* *}$ & -0.35 & -1.45 & $2.26^{*}$ \\
\hline RohinixTCGS-1416 & $-4.65^{* *}$ & $-7.66^{* *}$ & $-8.07^{* *}$ & $-1.83^{*}$ & $-2.90^{* *}$ & 0.75 \\
\hline NarayanixTCGS-1416 & $-10.75^{* *}$ & $-12.15^{* *}$ & $-12.15^{* *}$ & $-9.70^{* *}$ & $-10.37^{* *}$ & $-9.02^{* *}$ \\
\hline Narayanix|CGV-91114 & -1.38 & $-2.50^{* *}$ & $-5.61^{* *}$ & $4.20^{* *}$ & 1.11 & $2.63^{* *}$ \\
\hline NarayanixTMV-2 & $2.78^{* *}$ & $2.78^{* *}$ & -0.45 & $4.46^{* *}$ & $4.44^{* *}$ & $6.02^{* *}$ \\
\hline NarayanixDharani & $-9.41^{* *}$ & $-10.63^{* *}$ & $-11.03^{* *}$ & $-2.22^{* *}$ & $-2.22^{*}$ & -0.75 \\
\hline K-6×Dharani & $3.71^{* *}$ & 0.90 & 0.90 & $2.36^{* *}$ & -1.05 & $6.02^{* *}$ \\
\hline K-6×TCGS-1416 & $5.26^{* *}$ & $5.26^{* *}$ & -0.45 & $3.53^{* *}$ & $-2.11^{*}$ & $4.89^{* *}$ \\
\hline K-6xICGV-91114 & 1.19 & 0.00 & $-3.14^{* *}$ & $2.00^{*}$ & -0.7 & $6.39^{* *}$ \\
\hline $\mathrm{K}-6 \times \mathrm{TMV}-2$ & 0.25 & $-2.25^{* *}$ & $-2.69^{* *}$ & -0.18 & $-2.81^{* *}$ & $4.14^{* *}$ \\
\hline GreeshmaxDharani & 0.69 & -1.35 & -1.35 & 0.19 & 0.00 & 0.38 \\
\hline Greeshmax ICGV-91114 & $-2.09^{* *}$ & $-2.8^{* *}$ & $-6.73^{* *}$ & $4.41^{* *}$ & $1.87^{*}$ & $2.26^{*}$ \\
\hline GreeshmaxTCGS-1416 & $-6.05^{* *}$ & $-6.48^{* *}$ & $-9.42^{* *}$ & $2.44^{* *}$ & $1.89^{*}$ & $3.38^{* *}$ \\
\hline GreeshmaxTMV-2 & 0.46 & -1.35 & $-1.79^{*}$ & $3.54^{* *}$ & $2.96^{* *}$ & $4.51^{* *}$ \\
\hline SEm \pm & 0.53 & 0.61 & 0.61 & 0.73 & 0.84 & 0.84 \\
\hline
\end{tabular}

Table 1 Continue...

\begin{tabular}{|c|c|c|c|c|c|c|}
\hline \multirow[t]{3}{*}{ Crosses } & \multicolumn{6}{|c|}{ Heterosis (\%) } \\
\hline & \multicolumn{3}{|c|}{ Kernel yield plant ${ }^{-1}(\mathrm{~g})$} & \multicolumn{3}{|c|}{ Pod yield plant ${ }^{-1}(\mathrm{~g})$} \\
\hline & $>\mathrm{MP}$ & $>\mathrm{BP}$ & $>\mathrm{SP}$ & $>\mathrm{MP}$ & $>\mathrm{BP}$ & $>\mathrm{SP}$ \\
\hline TAG-24×ICGV-91114 & $26.8^{* *}$ & $18.3^{* *}$ & $18.30^{* *}$ & 20.67 & 13.16 & 29.26 \\
\hline TAG-24×TMV-2 & $16.61^{* *}$ & 6.55 & $-7.73^{*}$ & 4.76 & -11.23 & 1.40 \\
\hline TAG-24×TCGS-1416 & 5.01 & $-9.30^{* *}$ & $7.99^{*}$ & -2.48 & -7.81 & 18.24 \\
\hline TAG-24×Dharani & $93.55^{* *}$ & $90.75^{* *}$ & $70.10^{* *}$ & 69.57 & 57.37 & 79.76 \\
\hline Prasuna×Dharani & 0.72 & $-6.03^{*}$ & $8.51^{*}$ & 12.33 & 4.48 & 21.44 \\
\hline Prasuna× ICGV-91114 & $29.48^{* *}$ & 4.91 & $21.13^{* *}$ & 42.01 & 19.48 & 38.88 \\
\hline Prasuna×TMV-2 & 0.88 & -0.65 & $18.30^{* *}$ & 4.92 & 0.000 & 28.26 \\
\hline Prasuna×TCGS-1416 & $57.43^{* *}$ & $39.51^{* *}$ & $61.08^{* *}$ & 74.53 & 60.69 & 86.77 \\
\hline RohinixTMV-2 & $-41.92^{* *}$ & $-43.07^{* *}$ & $-40.72^{* *}$ & -43.31 & -46.84 & -39.28 \\
\hline RohinixICGV-91114 & $24.63^{* *}$ & 5.20 & $9.54^{* *}$ & 24.22 & 5.26 & 20.24 \\
\hline Rohinix Dharani & $-12.24^{* *}$ & $-17.75^{* *}$ & -2.06 & -10.74 & -15.63 & 8.22 \\
\hline Rohini× TCGS-1416 & $16.80^{* *}$ & $8.42^{*}$ & $12.89^{* *}$ & 24.57 & 15.61 & 32.06 \\
\hline NarayanixTCGS-1416 & 5.74 & -0.26 & -0.26 & -48.93 & $-67.32^{* *}$ & 16.83 \\
\hline Narayanix ICGV-91114 & $14.15^{* *}$ & 3.20 & $-8.51^{*}$ & -53.21 & $-71.41^{* *}$ & 2.20 \\
\hline NarayanixTMV-2 & $12.16^{* *}$ & -2.16 & $16.49^{* *}$ & -50.91 & $-66.65^{* *}$ & 19.24 \\
\hline NarayanixDharani & 1.16 & 0.87 & $-10.05^{* *}$ & $77.73^{* *}$ & 13.17 & $304.61^{* *}$ \\
\hline K-6 x Dharani & $-23.82^{* *}$ & $-25.00^{* *}$ & $-25.00^{* *}$ & -22.81 & -25.74 & -19.64 \\
\hline K-6×TCGS-1416 & $65.14^{* *}$ & $43.6^{* *}$ & $39.18^{* *}$ & 51.50 & 31.30 & 42.08 \\
\hline K-6xICGV-91114 & $-22.43^{* *}$ & $-29.65^{* *}$ & $-16.24^{* *}$ & -23.73 & -29.69 & -9.82 \\
\hline K-6×TMV-2 & -3.60 & $-7.45^{*}$ & $-10.31^{* *}$ & -5.06 & -9.63 & -2.20 \\
\hline
\end{tabular}




\begin{tabular}{|c|c|c|c|c|c|c|}
\hline \multirow[t]{3}{*}{ Crosses } & \multicolumn{6}{|c|}{ Heterosis (\%) } \\
\hline & \multicolumn{3}{|c|}{ Kernel yield plant $^{-1}(\mathrm{~g})$} & \multicolumn{3}{|c|}{ Pod yield plant $^{-1}(\mathrm{~g})$} \\
\hline & $>\mathrm{MP}$ & $>B P$ & $>\mathrm{SP}$ & $>M P$ & $>\mathrm{BP}$ & $>S P$ \\
\hline GreeshmaxDharani & $80.91^{* *}$ & $68.56^{* *}$ & $68.56^{* *}$ & -7.39 & -38.32 & 85.77 \\
\hline GreeshmaxICGV-91114 & $-10.93^{* *}$ & $-18.51^{* *}$ & $-29.64^{* *}$ & -58.82 & -73.99 & -21.64 \\
\hline Greeshma×TCGS-1416 & 1.13 & $-12.77^{* *}$ & 3.87 & -15.63 & -39.85 & 81.16 \\
\hline GreeshmaxTMV-2 & $67.99^{* *}$ & $65.32^{* *}$ & $47.42^{* *}$ & -23.96 & -49.63 & 51.70 \\
\hline SEm \pm & 0.38 & 0.43 & 0.43 & 10.59 & 12.23 & 12.23 \\
\hline
\end{tabular}

"Significant at $(p=0.05)$ level; ${ }^{* *}$ Significant at $(p=0.01)$ level ; MP: Mid parent; BP: Better parent; SP: Standard parent

Table 2: List of best $\mathrm{F}_{1} \mathrm{~s}$ for yield and water use efficiency traits for moisture stress tolerance in groundnut

\begin{tabular}{|c|c|c|c|}
\hline \multirow[t]{2}{*}{ Character } & \multicolumn{3}{|c|}{ Best heterotic crosses-based on } \\
\hline & Relative heterosis & Heterobeltiosis & Standard heterosis \\
\hline Plant height $(\mathrm{cm})$ & $\begin{array}{l}\text { TAG-24×TMV-2 } \\
\text { Rohini×Dharani } \\
\text { K-6×ICGV-91114 }\end{array}$ & $\begin{array}{l}\text { TAG-24×TMV-2 } \\
\text { Rohini×Dharani } \\
\text { K-6×ICGV-91114 }\end{array}$ & $\begin{array}{l}\text { TAG-24×TMV-2 } \\
\text { Rohini×Dharani } \\
\text { Rohini×TMV-2 }\end{array}$ \\
\hline $\begin{array}{l}\text { No. of primary branches } \\
\text { plant }^{-1}\end{array}$ & $\begin{array}{l}\text { GreeshmaxDharani } \\
\text { GreeshmaxICGV-91114 } \\
\text { GreeshmaxTCGS-1416 }\end{array}$ & $\begin{array}{l}\text { GreeshmaxDharani } \\
\text { GreeshmaxICGV-91114 } \\
\text { GreeshmaxTCGS-1416 }\end{array}$ & $\begin{array}{l}\text { GreeshmaxDharani } \\
\text { GreeshmaxICGV-91114 } \\
\text { GreeshmaxTCGS-1416 }\end{array}$ \\
\hline $\begin{array}{l}\text { No. of secondary branches } \\
\text { plant }^{-1}\end{array}$ & $\begin{array}{l}\text { NarayanixICGV-91114 } \\
\text { K-6×ICGV-91114 } \\
\text { NarayanixTMV-2 } \\
\text { K-6×Dharani }\end{array}$ & $\begin{array}{l}\text { NarayanixICGV-91114 } \\
\text { K-6×ICGV-91114 } \\
\text { NarayanixTMV-2 } \\
\text { K-6×Dharani }\end{array}$ & Prasuna×TCGS-1416 \\
\hline $\begin{array}{l}\text { SPAD chlorophyll meter } \\
\text { reading }\end{array}$ & $\begin{array}{l}\text { TAG-24×TMV-2 } \\
\text { K-6×TMV-2 } \\
\text { Greeshma×TMV-2 }\end{array}$ & $\begin{array}{l}\text { TAG-24×TMV-2 } \\
\text { K-6×TMV-2 } \\
\text { Greeshma×TMV-2 }\end{array}$ & $\begin{array}{l}\text { TAG-24×TMV-2 } \\
\text { K-6×TMV-2 } \\
\text { Greeshma×TMV-2 }\end{array}$ \\
\hline Specific leaf area $\left(\mathrm{cm}^{2} \mathrm{~g}^{-1}\right)$ & $\begin{array}{l}\text { TAG-24×ICGV-91114 } \\
\text { K-6×TMV-2 } \\
\text { K-6 xTCGS-1416 }\end{array}$ & $\begin{array}{l}\text { TAG-24×ICGV-91114 } \\
\text { K-6×TMV-2 } \\
\text { GreeshmaxTCGS-1416 }\end{array}$ & $\begin{array}{l}\text { TAG-24×ICGV-91114 } \\
\text { Greeshma×TCGS-1416 } \\
\text { Greeshma×Dharanl }\end{array}$ \\
\hline No. of mature pods plant ${ }^{-1}$ & $\begin{array}{l}\text { Prasuna×TCGS-1416 } \\
\text { TAG-24×Dharani } \\
\text { Greeshma×Dharani }\end{array}$ & $\begin{array}{l}\text { Prasuna×TCGS-1416 } \\
\text { TAG-24×ICGV-91114 } \\
\text { Greeshma×Dharani }\end{array}$ & $\begin{array}{l}\text { Prasuna×TCGS-1416 } \\
\text { TAG-24×Dharani } \\
\text { Greeshma×Dharani }\end{array}$ \\
\hline Shelling per cent & $\begin{array}{l}\text { Rohini×ICGV-91114 } \\
\text { K-6×TCGS-1416 } \\
\text { TAG-24×ICGV-91114 }\end{array}$ & $\begin{array}{l}\text { Rohini×ICGV-91114 } \\
\text { K-6×TCGS-1416 } \\
\text { TAG-24×Dharani }\end{array}$ & $\begin{array}{l}\text { Rohini×ICGV-91114 } \\
\text { TAG-24×Dharani } \\
\text { TAG-24×ICGV-91114 }\end{array}$ \\
\hline $\begin{array}{l}\text { Sound mature kernel per } \\
\text { cent }\end{array}$ & $\begin{array}{l}\text { Prasuna×Dharani } \\
\text { RohinixICGV-91114 } \\
\text { Rohini×TMV-2 }\end{array}$ & $\begin{array}{l}\text { Prasuna×Dharani } \\
\text { NarayanixTMV-2 } \\
\text { TAG-24×Dharani }\end{array}$ & $\begin{array}{l}\text { Prasuna×Dharani } \\
\text { Rohini×TMV-2 } \\
\text { Rohini×ICGV-91114 } \\
\text { TAG-24×Dharani }\end{array}$ \\
\hline Kernel yield plant ${ }^{-1}(\mathrm{~g})$ & $\begin{array}{l}\text { TAG-24×Dharani } \\
\text { Greeshma×Dharani } \\
\text { Greeshma×TMV-2 }\end{array}$ & $\begin{array}{l}\text { TAG-24×Dharani } \\
\text { Greeshma×Dharani } \\
\text { Greeshma×TMV-2 }\end{array}$ & $\begin{array}{l}\text { TAG-24×Dharani } \\
\text { Greeshma×Dharani } \\
\text { Prasuna×TCGS-1416 }\end{array}$ \\
\hline Pod yield plant $^{-1}(\mathrm{~g})$ & $\begin{array}{l}\text { Narayani×Dharani } \\
\text { Prasuna×TCGS-1416 } \\
\text { TAG-24×Dharani }\end{array}$ & $\begin{array}{l}\text { Prasuna×TCGS-1416 } \\
\text { TAG-24×Dharani } \\
\text { Prasuna×ICGV-91114 }\end{array}$ & $\begin{array}{l}\text { Narayani×Dharani } \\
\text { Prasuna×TCGS-1416 } \\
\text { Greeshma×Dharani }\end{array}$ \\
\hline
\end{tabular}

to $8.58 \%$ (RohinixICGV-91114). Four $F_{1} s$ recorded significant positive heterosis and twelve $\mathrm{F}_{1}$ s recorded significant negative heterosis over better parent. Standard heterosis ranged from
-12.56\% (PrasunaxTCGS-1416) to 2.69\% (TAG-24×Dharani). Only two $\mathrm{F}_{1} \mathrm{~s}$ recorded significant positive heterosis and seventeen $\mathrm{F}_{1} \mathrm{~s}$ recorded significant negative heterosis over 
standard parent. These results were confirmed with findings of John et al. (2012), John and Raghava Reddy (2015) and Waghmode et al. (2017).

\subsubsection{Sound mature kernel per cent}

The relative heterosis for sound mature kernel per cent varied from $-9.70 \%$ (NarayanixTCGS-1416) to $10.65 \%$ (PrasunaxDharani). Fifteen $\mathrm{F}_{1} \mathrm{~s}$ registered significant positive heterosis and four $\mathrm{F}_{1} \mathrm{~s}$ showed significant negative heterosis over mid parent. Heterobeltiosis ranged from $-10.37 \%$ (NarayanixTCGS-1416) to $10.04 \%$ (PrasunaxDharani). Ten $\mathrm{F}_{1} \mathrm{~S}$ recorded significant positive heterosis and eight $F_{1} s$ recorded significant negative heterosis over better parent. Standard heterosis ranged from $-9.02 \%$ (NarayanixTCGS-1416) to $11.28 \%$ (PrasunaxDharani). Seventeen $\mathrm{F}_{1}$ s recorded significant positive heterosis and only two $\mathrm{F}_{1} \mathrm{~s}$ recorded significant negative heterosis over standard parent.

\subsubsection{Kernel yield plant ${ }^{-1}(g)$}

The range of heterosis over mid-parent varied from $-41.92 \%$ (RohinixTMV-2) to $93.55 \%$ (TAG-24×Dharani). Out of twenty four $F_{1} s$, twelve $F_{1} s$ recorded significant positive heterosis and five $\mathrm{F}_{1} \mathrm{~S}$ showed significant negative heterosis over midparent. Heterobeltiosis ranged from -43.07\% (RohinixTMV-2) to $90.75 \%$ (TAG-24×Dharani). Significant and positive heterosis was observed in seven $\mathrm{F}_{1} \mathrm{~s}$. Significant negative heterosis was noticed in nine $\mathrm{F}_{1}$ s. Standard heterosis ranged from $-40.72 \%$ (RohinixTMV-2) to 70.10\% (TAG-24×Dharani). Out of twenty four $F_{1} s$, twelve $F_{1} s$ recorded significant positive heterosis and non significant positive heterosis was noticed in eight $F_{1}$ s over standard parent. Similar results were reported by John et al. (2012), John et al. (2014), and John and Raghava Reddy (2015).

\subsubsection{Pod yield plant ${ }^{-1}(g)$}

Relative heterosis ranged from $-50.91 \%$ (NarayanixTMV-2) to $77.73 \%$ (NarayanixDharani). Only one $\mathrm{F}_{1}$ recorded significant positive heterosis over mid parent. Heterobeltiosis ranged from -66.65\% (NarayanixTMV-2) to 60.69\% (PrasunaxTCGS-1416). Three $F_{1} s$ registered significant negative heterobeltiosis. Standard heterosis ranged from $-39.28 \%$ (RohinixTMV-2) to $304.61 \%$ (NarayanixDharani). Only one $F_{1}$ recorded significant heterosis over standard parent. Heterosis for pod yield in groundnut was also reported by Deshmukh (1985), Reddi et al. (1989), Bansal et al. (1993), Varman and Raveendran (1997), Rudraswamy et al. (1999), Parmar et al. (2004), Sharma and Gupta (2008), Jivani et al. (2009), John et al. (2012), John et al. (2014); John and Raghava Reddy (2015).

\section{Conclusion}

The crosses, TAG-24×TMV-2 and PrasunaxDharani had recorded with high relative heterosis, beter parent heterosis and standard parent heterosis for SPAD chlorophyll meter reading and sound mature kernel per cent, TAG$24 \times$ ICGV-9114 with low relative heterosis, better parent heterosis and standard parent heterosis for specific leaf area, and NarayanixDharani with high relative heterosis and standard parent heterosis for pod yield plant ${ }^{-1}$. These hybrids offer best possibilities of future exploitation for development of high yielding moisture stress tolerant genotypes.

\section{Acknowledgement}

This research was supported under the UGC Research award to the first author by the University Grants Commission (UGC), New Delhi is gratefully acknowledged.

\section{References}

Arunachalam, V., Bandopadhyay, A., Nigam, S.N., Gibbons, R.W., 1984. Heterosis in relation to genetic divergence and specific combining ability in groundnut (Arachis hypogaea L.). Euphytica 33, 33-39.

Babitha, M., Reddy, P.V., 2001. Physiological traits related to water use efficiency in groundnut under stress and non- stress conditions. Journal of Research 29(1), 9-15.

Bansal, V.K., Satiya, P.R., Gupta, V.P., Sangh, A.S., Verma, M.M., 1993. Heterosis in relation to plant type in groundnut for yield in heterosis breeding. In: Crop Plants Theory and Application, Virk, D.S., Chahal, G.S. (Eds.), Symposium, Ludhiana 23-24, Feb. 1993, 6-7.

Bhagsari, A.S., Brown, R.H., 1976. Photosynthesis of peanut (Arachis hypogaea L.) genotypes. Peanut Science 3, 1-9.

Briggs, L.J., Shantz, 1913. The water requirements of plants II. A review of the literature. Journal of Agricultural Research 3, 1-64.

Deshmukh, S.K., Zade, V.R., Reddy, P.S., 1985. Heterobeltiosis in groundnut. Indian Journal of Agricultural Science 85, 358-361.

Hammond, L.C., Boote, K.J., 1981 Water use efficiency of peanuts. Proceedings, American Peanut Research and Education Society 13, 109.

Hebbar, K.B., 1990. Studies on genotypic variation in water use efficiency in groundnut and its relevance to productivity under field condition. M.Sc. (Agri.) Thesis, submitted to University of Agricultural Sciences, Bangalore.

Hebbar, K.B., Sashidhar, V.R., Udayakumar, M., Devendra, R., Rao, R.C.N., 1994. A comparative assessment of water use efficiency in groundnut (Arachis hypogaea) grown in containers and in field under water limited conditions. Journal of Agricultural Sciences 122, 249-434.

Hubick, K.T., Farquhar, G.D., Shorter, R., 1986. Correlation between water use efficiency and carbon isotope discrimination in diverse peanut (Arachis) germplasm. Australian Journal of Plant Physiology 13, 803-816.

Jivani, L.L., Khanpara, M.D., Kachhadia, V.H., Vacchani. J.H., 2009. Combining ability for pod yield and its components in groundnut (Arachis hypogaea L.) International Journal of Agriculture Sciences 5(1), 248-250.

John, K., Raghava Reddy, P., 2015. Heterosis and inbreeding depression for yield and yield attributes in groundnut (Arachis hypogaea L.). International Journal of Current Research in Biosciences and Plant Biology 2(7), 135-148. 
John, K., Raghava Reddy, P., Haripraad Reddy, K., Sudhakar, P., Eswar Reddy., N.P., 2012. Estimation of heterosis for certain morphological, yield and yield attributes in groundnut (Arachis hypogaea L.). Legume Research 35(3), 194-201.

John, K., Raghava Reddy, P., Hariprasad Reddy, K., Sudhakar, P., Eswar Reddy, N.P., 2012. Identification of best heterotic crosses for yield and water use efficiency traits in groundnut (Arachis hypogaea L.). Journal of Plant Breeding and Crop Science Vol. 4(2), 17-24.

John, K., Reddy, R.P., 2014. Combining ability and heterosis for yield and water use efficiency traits in groundnut. Legume Research-An International Journal 373, 235244.

John, K., Raghava Reddy, P., Hariprasad Reddy, K., Sudhakar, P., Eswar Reddy, N.P., 2014. Studies on Heterosis and inbreeding depression for yield and phsiological traits in groundnut (Arachis hypogaea L.). Legume Research - An International Journal, 37(2), 117-125.

Nimitr, V., Sirichai, C., Somyot, D., 1988. Root growth and yield of groundnut under different soil conditions in North East Thailand. In Proceedings of the Sixth Thailand National Groundnut Meeting, Songkhla, Thailand, 334-343.

Pallas, J.E ., Samish, Y.B., 1974. Photosynthetic response of peanut. Crop Science 14, 478-482.

Pallas, J.E., 1982. Photosynthetic traits of selected peanut genotypes. Peanut Science 9, 14-17.

Parmar, D.L., Rathnakumar, L., Bharodia, P.S., Dobaria, J.R., 2004. Genetic basis of heterosis in crosses involving adapted and exotic groundnut germplasm. Paper Presented at the National Symposium on Enhancing Productivity of Groundnut for Sustaining Food and Nutritional Security, 11-13 October-2004 at NRCG, Junagadh.

Reddi, M.V., Reddy, K.R., Reddy, K.C.M., Reddy, K.H.P., Reddy, P.R., Reddy, G.L.K., 1989. Heterosis and combining ability in $6 \times 6$ diallel set of groundnut (Arachis hypogaea L.). Journal of Research APAU 17, 378-383.

Rosario, D.A., Fajardo, F.F., 1988 Morphophysiological responses to water stress of ten varieties of peanut (Arachis hypogaea L.) Phillippine Journal of Crop Science 13, Supplement I, Field Crop Abstracts 42, 8.

Rudraswamy, P., Nehru, S.D., Kulkarni, R.S., Manjunath, A., 1999. Estimation of genetic variability and inbreeding depression in six crosses of groundnut (Arachis hypogaea L.). Mysore journal of agricultural sciences 33(2), 248-252.

Seethala Devi, G., 2004. Genetic studies on certain morphological and physiological attributes in $10 \mathrm{~F}_{2}$ populations of groundnut (Arachis hypogaea L.) M.Sc. (Ag.) Thesis, Acharya N.G. Ranga Agricultural University, Hyderabad.

Tanner, C.B., Sinclair, T.R., 1983 Efficient water use in crop production. Research or Re-search ? In: Taylor, H.M., Jordon, I.R., Sinclair, T.R. (Eds.), Limitations to efficient water use in crop production. American Society of Agronomy Crop Science. Society of America and Soil Science Society of America 1-27.

Udayakumar, M., Sheshshayee, M.S., Nataraj, K.N., Bindu Madhava, H., Devendra, R., Aftab Hussain, I.S., Prasad, V.T.G., 1998. Why has breeding for WUE not been successful? An analysis and alternate approach to exploit this trait for crop improvement. Current Science 74, 994-1000.

Varman, P.V., Raveendran, T.S., 1997. Comparison of single and three way crosses in groundnut. Madras Agricultural Journal 84(2), 70-73.

Waghmode, B.D., Navhale, V.C. , Sonone, N.G., Thawre, B.L., 2017. Linextester analysis for heterosis in groundnut (Arachis hypogaea L.). Electronic Journal of Plant Breeding 8(1), 347-355.

Wright, G.C., Hubick, K.T., Farquhar, 1988. Discrimination in carbon isotopes of leaves correlates with water use efficiency of field grown peanut cultivars. Australian Journal of Plant Physiology 15, 815-825.

Wright, G.C., Bell, M.J., 1992. Plant population studies in peanut (Arachis hypogaea L.) in sub tropical Australia in water limited conditions. Australian Journal of Experimental Agriculture 32, 189-196.

Wright, G.C., Sarwanto, T.A., Rahmiana, A., Syarifuddin, A., 1992. Investigation of drought tolerance traits conferring adaptation to drought stress in peanut. 74-84. ACIAR Proceedings No. 40, Canberra, Australia.

Wright, G.C., Kerry Hubick, K.T., Farquhar, G.D., Nageswara Rao, R.C., 1993. Genetic and Environmental Variation in transpiration efficiency and its correlation with carbon isotope discrimination and specific leaf area in peanut p. 247-267 In: J. Ehleringer et al. (ed.). "Stable isotopes and plant carbon water relations" Academic Press San Diego. CA. 